\title{
Robust and Flexible Tracking of Vehicles Exploiting Soft Map-Matching and Data Fusion
}

\author{
Marouan Mizmizi*†, Silvio Mandelli ${ }^{\dagger}$, Stephan Saur ${ }^{\dagger}$, Luca Reggiani* \\ *Politecnico di Milano, 20133 Milano, Italy E-mail: \{marouan.mizmizi, luca.reggiani\}@polimi.it \\ ${ }^{\dagger}$ Nokia Bell Labs, 70435 Stuttgart, Germany E-mail: \{silvio.mandelli, stephan.saur\}@ nokia-bell-labs.com
}

\begin{abstract}
Accurate positioning of vehicles and pedestrians is crucial for enhancing road safety. In this paper we propose and compare two implementations based on Unscented Kalman Filter (UKF) and Particle Filter (PF) to perform trajectory estimation with sensor fusion. For the latter, a novel soft map-matching technique is applied on top of a Particle Filter. The main benefit of our proposal is the possibility of detecting reliably critical situations, like vehicles skidding off the road. Moreover, we can reduce the positioning error by $45 \%$ w.r.t. a prior art approaches. Our solution can be implemented as a cloud service in the 5G mobile radio network.
\end{abstract}

Index Terms-5G, V2X, autonomous driving, Kalman filter, Particle filter, road safety, map-matching.

\section{INTRODUCTION}

The first pedestrian victim of an automobile collision occurred in 1896 [1]. Since then, a huge effort has been invested to improve road safety, both for vehicles and Vulnerable Road Users (VRUs), like pedestrians and cyclists. However, this work began to bring considerable results only in the last decade, where the number of road traffic deaths - 1.25 million in 2013 - has began to plateau since 2007 despite the global increase in population and motorization [2]. Designing a system for protecting people traveling in streets with cars, motorcycles, bikes and by foot is an extremely difficult task as position and trajectory of the users have to be recovered with high precision and without interruptions. This goal can be achieved by merging together data coming from many sensors, systems, and fusing it together with a-priori knowledge of the scenario, e.g. the road map. Our work focuses on this target, that is fusing data we have at our disposal at each time instant to get the best vehicle trajectory estimate possible.

Today, localization relies mostly on Global Navigation Satellite Systems (GNSS), like the Global Positioning System (GPS), which can be complemented by onboard equipments of vehicles as cameras, radar and other sensors [3]. GPS works well in open areas with unhindered Line-of-Sight links to a sufficient number of satellites. However, accurate GPS localization fails in tunnels, under bridges, in parking garages [4], while inappropriate weather conditions and obstacles, as other cars and buildings, can limit performance of video [5]. Alternatively, the radio access network can be utilized for obtaining localization as well. 3GPP Long Term Evolution (LTE) supports the Observed Time Difference of Arrival (OTDoA) measurements of Positioning Reference Signals (PRS) sent out from the LTE Base Station (eNB). The tracked user (TU) can either determine its position on its own with a multi-lateration algorithm, or it could report the OTDoA measurements to a network entity taking over this task. However, the localization accuracy is mainly limited by the Non-Line-of-Sight propagation conditions of the PRS between eNB and TU, leading to a bias in the OTDoA measurements. This effect can be compensated partially by advanced methods like the Blind Learning Algorithm for channel bias Distribution Estimation (BLADE) [6]. Nevertheless, even for perfectly synchronized BSs, the achievable localization accuracy can hardly fulfill the stringent performance requirements throughout all propagation conditions and deployment scenarios. Then, during vehicle tracking, map matching is used to identify the actual road on which the TU is moving. During the last years, many solutions for map matching have been proposed: probabilistic approaches [7], fuzzy logic [8], advanced techniques [9] and particle filters with weights modified according to position and velocity in the map topology [10].

In this paper, we present an integrated solution to solve the trajectory tracking problem based on the considerations above, exploiting the recursive Bayesian estimation theory. First, we implement and compare two tracking solutions, i.e. the Unscented Kalman Filter (UKF) and the Particle Filter (PF), and then we focus on an enhanced PF implementation by introducing a novel map-matching technique. All the previous map-matching approaches are based on the assumption that the vehicle is on a road segment. Here we target critical situations, when the driver loses control of the vehicle and drives off the road, thus there is the need to relax the constraint of vehicles being strictly on the road segments. Our PF algorithm applies soft weights to particles corresponding to positions outside the road, allowing a flexible solution that is quick to react to emergencies.

Performance of our approach is validated in a real-world scenario, providing performance and design insight on these tracking solutions. The remainder of the paper is organized as follows: Section II presents the system overview and describes the recursive Bayesian estimation. In Section III, we formalize the map-matching approach and, in Section IV, we summarize the most relevant numerical results. Conclusions will be drawn in Section V.

\section{SYSTEM MODEL}

The proposed tracking system can be implemented in a mobile radio network, eventually as a cloud service. It can rely on different inputs, as illustrated in Fig. 1. The main source of information is the position estimation based on 


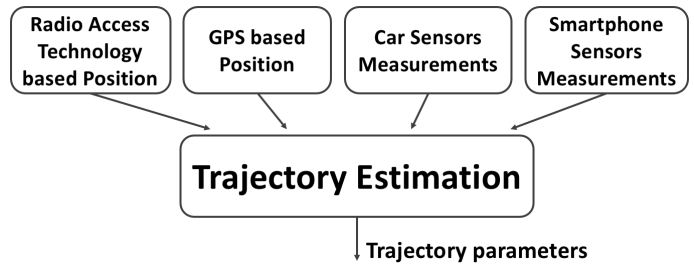

Fig. 1: Fusion of different information sources.

OTDoA measurements from the Radio Access Technology (RAT) network and BLADE [6]. Other inputs might be used when they are available, like GPS and car or smartphone sensors. The results of our trajectory estimation algorithm are the trajectory's parameters, e.g., current position, speed, heading and turn rate. In this work, we assume that the current position is the key performance indicator of interest and we compare different schemes through their respective position error distributions and average values. In the next subsection, we provide a brief review of the Bayesian techniques used for tracking.

\section{A. Tracking as a Recursive Bayesian State Estimation}

Determining the unknown state of a dynamic system using noisy and distorted observations is the subject of recursive Bayesian theory [11]. In our particular case, the dynamic system is a moving vehicle or a VRU. The state variables are the position, velocity, heading, and turn rate. Let us consider the evolution of the unknown state vector sequence $\mathbf{x}_{k}$ of a $\mathrm{TU}$ given by

$$
\mathbf{x}_{k}=\hat{\mathbf{f}}_{k}\left(\mathbf{x}_{k-1}, \mathbf{v}_{k-1}\right)
$$

where $\hat{\mathbf{f}}_{k}: \mathbb{R}^{d_{x}} \times \mathbb{R}^{d_{v}} \rightarrow \mathbb{R}^{d_{x}}$ is a generic function of the state $\mathbf{x}_{k-1}$, while $\mathbf{v}_{k-1}$ is an independent and identically distributed (i.i.d.) process noise sequence, $d_{x}, d_{v}$ are dimensions of the state and process noise vectors, respectively. The state vector $\mathbf{x}_{k}$ includes a set of variables that describe the status of the system, and it is the variable we want to track. It evolves at discrete times $k$ as a result of the assumptions made for the dynamic state model of Eq. (3). Then, the purpose of tracking is to determine recursively the probability density function (pdf) of $\mathbf{x}_{k}$ from the measures

$$
\mathbf{y}_{k}=\hat{\mathbf{h}}_{k}\left(\mathbf{x}_{k}, \mathbf{n}_{k}\right)
$$

where $\hat{\mathbf{h}}_{k}: \mathbb{R}^{d_{x}} \times \mathbb{R}^{d_{n}} \rightarrow \mathbb{R}^{d_{y}}$ is a generic function, $\mathbf{n}_{k}$ is an i.i.d. measurement noise sequence, and $d_{y}, d_{n}$ are the dimensions of the measurement and measurement noise vectors, respectively.

The Bayesian approach recursively estimates the pdf of the state $\mathbf{x}_{k}$ at time $k$, given all the measurement data $\mathbf{y}_{1: k}$ from the first step to step $k$. Accordingly, the estimate of $p\left(\mathbf{x}_{k} \mid \mathbf{y}_{1: k}\right)$ is done in two stages, predict and update. An exhaustive description of this techniques is given in [11] and [12].

Hereinafter, the process noise $\mathbf{v}_{k}$ and the measurement noise $\mathbf{n}_{k}$ are assumed to be additive and independent Gaussian terms

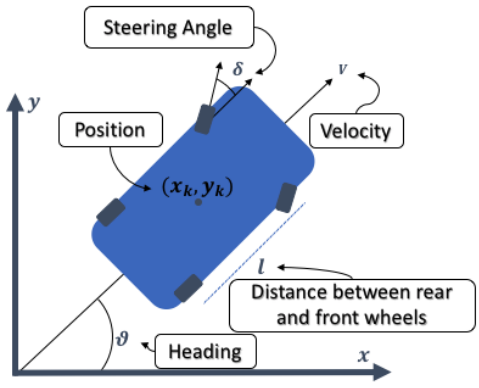

Fig. 2: State parameters description.

[12], the state dynamic model in (3) and the measurement model in (4) become

$$
\begin{array}{r}
\mathbf{x}_{k}=\mathbf{f}_{k}\left(\mathbf{x}_{k-1}\right)+\mathbf{v}_{k-1} \\
\mathbf{y}_{k}=\mathbf{h}_{k}\left(\mathbf{x}_{k}\right)+\mathbf{n}_{k}
\end{array}
$$

where $\mathbf{f}_{k}$ and $\mathbf{h}_{k}$ are generic functions that describe the deterministic component of the two models, and $\mathbf{v}_{k-1}$ and $\mathbf{n}_{k}$ are

$$
\begin{aligned}
\mathbf{v}_{k-1} & \sim \mathcal{N}\left(0, \mathbf{Q}_{k-1}\right) \\
\mathbf{n}_{k} & \sim \mathcal{N}\left(0, \mathbf{R}_{k}\right),
\end{aligned}
$$

where $\mathbf{Q}_{k-1} \in \mathbb{R}^{d_{v}}$ is the covariance matrix of the process noise, and $\mathbf{R}_{k} \in \mathbb{R}^{d_{n}}$ is the covariance matrix of the measurement noise.

In the next subsection we describe two different dynamic models.

\section{B. Dynamic State Model}

Defining a proper dynamic state model and tuning its parameters is one of the biggest challenges in the design of a tracking system. Factors such as driver habits or external contingencies make the prediction of the TU behavior really a hard task [13]. A comparison of different dynamic models for target tracking is given in [14]. The two models considered here are described in the next paragraphs. The Constant Velocity model (CV) has less modeling power compared to the Constant Turn Rate and Velocity (CTRV). namely less tracked "constant" quantities. Depending on the user behavior and conditions one can be more suited than the other, and the switching technique between the two will be object of future work. The tradeoff between CV and CTRV can be easily explained by noticing that the former can underfit the measurements, not being able to capture well constant and long turning trajectories, while the latter can overfit the noisy measurements if the trajectory is rectilinear.

1) CV Model: it is a linear model and the linear state transition is

$$
\mathbf{x}_{k+1}=\left(\begin{array}{c}
x_{k}+\Delta T v_{y k} \\
v_{y k} \\
y_{k}+\Delta T v_{y k} \\
v_{y k}
\end{array}\right)
$$


where $\mathbf{x}_{k}=\left\{x_{k}, v_{x}, y_{k}, v_{y}\right\}$ is the state space, $x, y$ are the position coordinates of the $\mathrm{TU}, v_{x}, v_{y}$ are the constant velocities in both dimensions.

2) CTRV Model: the state space $\mathbf{x}_{k}=\left\{x_{k}, y_{k}, \theta, v, \omega\right\}$ can be transformed by the non-linear state transition:

$$
\mathbf{x}_{k+1}=\left(\begin{array}{c}
x_{k}+\frac{v_{k}}{\omega_{k}} \sin \left(\omega_{k} \Delta T+\theta_{k}\right)-\frac{v_{k}}{\omega_{k}} \sin \left(\theta_{k}\right) \\
y_{k}-\frac{v_{k}}{\omega_{k}} \cos \left(\omega_{k} \Delta T+\theta_{k}\right)+\frac{v_{k}}{\omega_{k}} \sin \left(\theta_{k}\right) \\
\omega_{k} \Delta T+\theta_{k} \\
v_{k} \\
\omega_{k}
\end{array}\right)
$$

where $x, y$ are the coordinates, $\theta$ is the heading, $v$ is the absolute value of the velocity and $\omega=v / l \cdot \tan (\delta)$ is the turn rate. The relation between these variables and the physical properties of the vehicle are illustrated in Fig. 2.

\section{Measurement Model}

The core of our algorithm regards the estimation of the target position, based on the OTDoA measurements. However, if other sources, such as GPS, car sensors and smartphone sensors are available, the system is able to use them in order to improve the accuracy of the estimate. To make this possible, it is necessary to make the measurement model in (4) time variant to accept at each time a different set of sources. Consequently, the dimensions $d_{y}$ and $d_{n}$ change over the time according to available measurements.

For instance, if at time $k$ only the position estimated trough the OTDoA measurements is available, the measurement model will be

$$
\mathbf{y}_{k}=\mathbf{h}_{k}\left(\mathbf{x}_{k}, \mathbf{n}_{k}\right)=\left(\begin{array}{c}
\left.h_{k, \mathrm{X} \text {-OTDOA }}\left(x_{k}\right)+n_{k, 1}\right) \\
h_{k, \text { Y-OTDOA }}\left(y_{k}\right)+n_{k, 2}
\end{array}\right)
$$

However, if at time $k+1$, we get also GPS and velocity measurements from the car sensors, the measurement model becomes

$$
\mathbf{y}_{k+1}=\left(\begin{array}{c}
h_{k+1, \mathrm{X}-\mathrm{OTDOA}}\left(x_{k}\right)+n_{k+1,1} \\
h_{k+1, \mathrm{Y} \text {-OTDOA }}\left(y_{k}\right)+n_{k+1,2} \\
h_{k+1, \mathrm{X}-\mathrm{GPS}}\left(x_{k}\right)+n_{k+1,3} \\
h_{k+1, \mathrm{Y}-\mathrm{GPS}}\left(y_{k}\right)+n_{k+1,4} \\
h_{k+1, \text { Speed }}\left(v_{k}\right)+n_{k+1,5}
\end{array}\right)
$$

where $h_{i}$ is a non-linear function that describes the relation between the measured quantity and the measurement.

\section{Tracking Algorithm}

We compared two algorithms for tracking, the Unscented Kalman Filter (UKF) and the Particle Filter (PF). The former is a parametric estimation of the posterior pdf and it is based on the so-called "unscented transform", which samples the pdf in determined points in order to track the mean and covariance of the state variable passing through nonlinear transformations [15]. The latter is a non-parametric estimation, which represents the required posterior pdf by a set of random samples of the state-space $\mathbf{x}_{k}^{(i)}$ (the $i$-th particle at time $k$ ) with the associated weights $w_{k-1}^{(i)}$ [12].
In terms of computational complexity, UKF outperforms PF since it has to track only a limited number of parameters while PF tracks $N_{s}$ particles, where $N_{s}$ depends on the application and the target accuracy. Moreover, PF suffers from the curse of dimensionality [12], which means that the complexity increases exponentially with the number of variables in the state vector $\mathbf{x}_{k}$.

\section{Soft MAP-MATCHING}

Map-matching can improve the tracking performance through a more accurate positioning by exploiting a-priori road map knowledge. This is done by assuming that vehicles typically follow the road path. Including road-map information within the common Kalman filtering framework represents a hard challenge due to the fact that road-maps express a step function behavior, that is highly non-linear, non-derivable, and thus critical w.r.t. the typical assumptions of Kalman filters. Therefore, we limit the map-matching implementation only to $\mathrm{PF}$, which is not subject to these constraints and is also able to capture efficaciously multi-modal pdf.

In our implementation, map information is embedded by changing softly the likelihood applied during the update phase of the PF. First, the state space is increased by a map flag $s_{k}$ that indicates in which area of the map the target is currently located, i.e.

$$
\breve{\mathbf{x}}_{k}=\left\{\mathbf{x}_{k}, s_{k}\right\}
$$

where $\mathbf{x}_{k}$, is the state variable defined by the state transition models in (7) and (8), and $s_{k}$ is defined as

$$
s_{k}= \begin{cases}0 & \text { if } \mathbf{x}_{k} \text { belongs to a road segment } G \\ 1 & \text { if } \mathbf{x}_{k} \text { belongs to zone } N \\ 2 & \text { if } \mathbf{x}_{k} \text { belongs to zone } F\end{cases}
$$

where zone $N$ is the area close to a road segment, and zone $F$ is the area far from a road segment. Formally, we define the zone $N=\left\{\left(x_{p}, y_{p}\right):\left(x_{p}, y_{p}\right) \notin G \wedge d_{p} \leq D\right\}$ and the zone $F=\left\{\left(x_{p}, y_{p}\right):\left(x_{p}, y_{p}\right) \notin G \wedge d_{p}>D\right\}$, where $d_{p}$ is the Euclidean distance between $\left(x_{p}, y_{p}\right)$ and the closest road segment and $D$ is a threshold, e.g. the sidewalk width.

Without loss of generality, we choose $s_{k} \in \mathcal{L}=\{0,1,2\}$ for our application; however, more labels can be defined in $\mathcal{L}$ if needed. In Fig. 3, an example of different zones is illustrated: it is obtained with OpenStreet Maps [16] and it corresponds to the roads of the Bell Labs campus in Stuttgart shown in Fig. 4.

In our PF implementation, the prediction step is done as defined in [12]. Given the $i$-th particle state $\mathbf{x}_{k-1}^{(i)}$ at time $k$, the new predicted state $\mathbf{x}_{k}^{(i)}$ is

$$
\mathbf{x}_{k}^{(i)}=\mathbf{f}\left(\mathbf{x}_{k-1}^{(i)}\right)+\mathbf{v}_{k-1}^{(i)}
$$

where $\mathbf{f}$ is the dynamic model in (7) and (8) and $\mathbf{v}_{k-1}^{(i)}$ is the process noise vector. Without map-matching, in the update step, the weight associated to the $i$-th particle is computed using the likelihood function of the measurements $p\left(\mathbf{y}_{k} \mid \mathbf{x}_{k}^{(i)}\right)$

$$
w_{k}^{(i)} \propto w_{k-1}^{(i)} p\left(\mathbf{y}_{k} \mid \mathbf{x}_{k}^{(i)}\right) .
$$




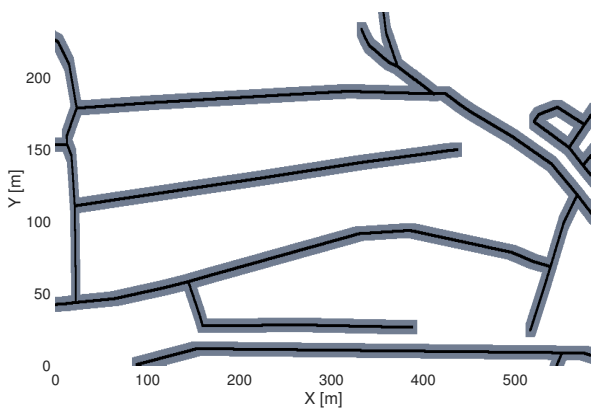

Fig. 3: Example of map zones. The center of the road is black, zones $N$ and $F$ are represented in blue and white respectively.

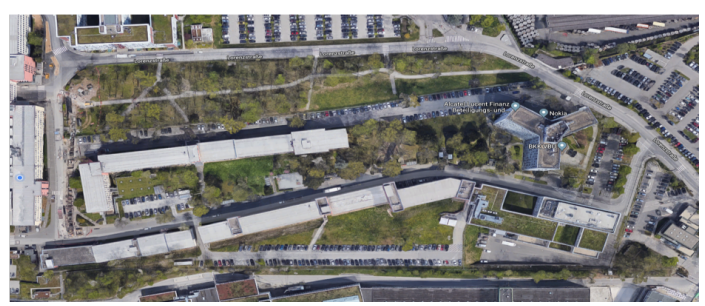

Fig. 4: Top view of the Nokia Bell Labs campus in Stuttgart.

\begin{tabular}{|c|c|c|c|c|}
\hline & \multicolumn{3}{|c|}{$\mathbf{s}_{k}$} \\
\hline & & 0 & 1 & 2 \\
\hline \multirow{3}{*}{$\mathbf{s}_{k-1}$} & 0 & 1 & 0.5 & 0.1 \\
\hline & 1 & 2 & 1 & 0.2 \\
\hline & 2 & 10 & 5 & 1 \\
\hline
\end{tabular}

TABLE I: Map-matching rules considered in the paper.

On the contrary, our solution introduces prior information based on map-matching and the update step becomes

$$
w_{k}^{(i)} \propto w_{k-1}^{(i)} p\left(\mathbf{y}_{k} \mid \mathbf{x}_{k}^{(i)}\right) M_{k}^{(i)}\left(s_{k}, s_{k-1}\right),
$$

where $M_{k}^{(i)}\left(s_{k}, s_{k-1}\right)$ is a parameter depending on the map flags $s_{k}^{(i)}$ and $s_{k-1}^{(i)}$ for the $i$-th particle. In our work, we assign values to $M_{k}^{(i)}\left(s_{k}, s_{k-1}\right)$ according to the rules defined in Table I. The values in Table I have been selected for a satisfactory performance result.

The particles are free to move across the map and, if a particle does not change the zone, i.e. $\left(s_{k}=s_{k-1}\right)$, its related weight is updated according to (14) $\left(M_{k}^{(i)}=1\right)$. However, if a particle changes the zone $\left(s_{k} \neq s_{k-1}\right)$, its corresponding weight is adapted according to (15). This definition is necessary in order to preserve the Markovian property for the state transition conditioned to the previous realization, and in order to avoid an exponential effect on the weights due to continuous multiplications if a particle falls outside the road. The idea is clear: if a particle leaves the street its likelihood gets penalized, and vice versa.

\section{Simulation Results}

In this Section we present simulation results for validating the performance of the proposed algorithm. The road network is loaded from the free database OpenStreetMap [16]. Vehicle trajectory is generated according to the maps and on the Bézier

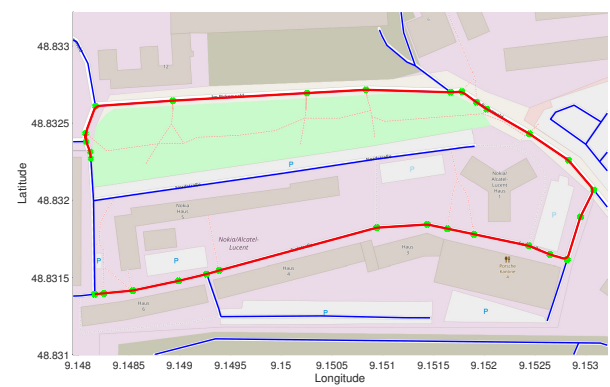

Fig. 5: Map view of the simulation scenario 1.

curves [17] and vehicle position measurements are simulated in Matlab.

Two scenarios have been evaluated by simulation. In the former in Fig. 5, the vehicle is assumed traveling along the roads shown in Fig. 3, while, in the latter in, a critical situation, in which the vehicle skids off the road, is emulated. The following assumptions are valid in all the simulations unless specified differently:

- OTDoA measurements are available each $200 \mathrm{~ms}$ and their standard deviation is $20 \mathrm{~ns}$;

- GPS measurements are available each $1 \mathrm{~s}$ and their standard deviation is $5 \mathrm{~m} \mathrm{[5]}$;

- PF resampling strategy is Systematic [12], threshold $\hat{N}_{\text {eff }}<0.3 N_{s}$ and number of particles is $N_{s}=5000$;

- The maximum velocity is $40 \mathrm{~km} / \mathrm{h}$ in straight roads and $20 \mathrm{~km} / \mathrm{h}$ along curves.

The choice of $N_{s}$ depends on the tradeoff between position accuracy and PF computational complexity. Our choice is justified since we have observed no substantial increase in accuracy when using $N_{s}>5000$, and that the average computational time to obtain an estimate is approximately 100 $\mathrm{ms}$, less than our measurements update rate. Note that UKF requires typically 3 order of magnitude less execution time, however doesn't allow to adopt map matching techniques, making it a good c̈heapšolution when limited in computation capacity.

\section{A. Scenario 1}

The results in Figs. 6 and 7 show that both algorithms, PF and UKF, improve the accuracy w.r.t single point position measurements, and map-matching applied to PF clearly outperforms the other solutions reducing the error by roughly $45 \%$ with both CV and CTRV models.

Table II summarizes the average positioning error for the two different dynamic models and tracking systems: the CV model in (7) shows better results in our scenarios. This effect becomes even more clear with UKF, where the average error achieved in the $\mathrm{CV}$ case is $2.46 \mathrm{~m}$. Notice that, with the CTRV model, the error achieves $3.47 \mathrm{~m}$, which is approximately $30 \%$ higher and this becomes less evident with PF. The reason of this difference is that the scenario is well described by the linear CV model in (7). The non-linear CRTV model in 


\begin{tabular}{|c|c|c|c|}
\cline { 2 - 4 } \multicolumn{1}{c|}{} & UKF & PF & PF with Map-matching \\
\hline CTRV & $3.47 \mathrm{~m}$ & $3.02 \mathrm{~m}$ & $1.66 \mathrm{~m}$ \\
\hline CV & $2.46 \mathrm{~m}$ & $2.58 \mathrm{~m}$ & $1.42 \mathrm{~m}$ \\
\hline \hline
\end{tabular}

TABLE II: Average positioning error in scenario 1.

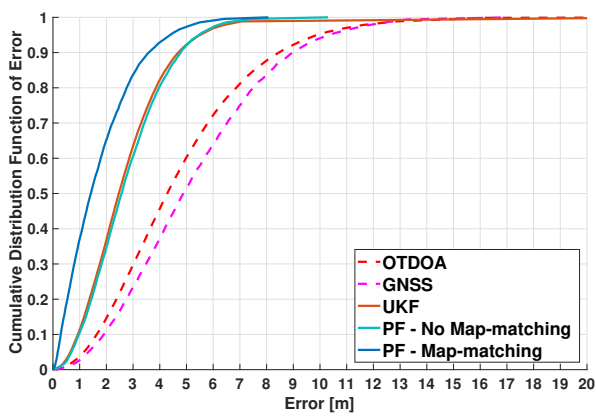

Fig. 6: Error CDF with the CV model.

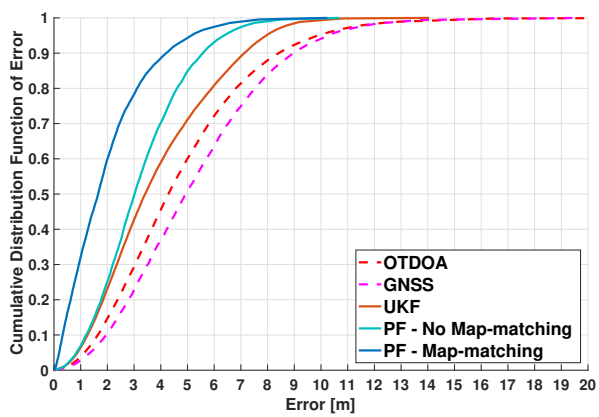

Fig. 7: Error CDF with CTRV model.

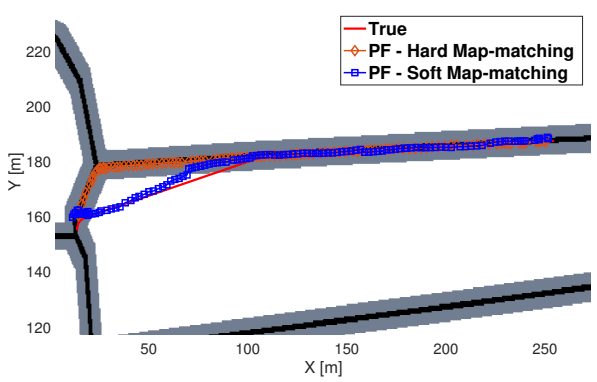

Fig. 8: Example of estimated trajectories in scenario 2.

(8) loses performance due to the over-fitting problem since it contains too many parameters w.r.t. data.

\section{B. Scenario 2}

In this scenario, we compare our proposed soft technique with a hard map-matching technique, where the weight of a particle outside the street is strictly zero. Notice from Fig. 8 that when the user does not follow the street, the hard map-matching is not able to track it. In this scenario, we have obtained an average error of $3.17 \mathrm{~m}$ for soft map-matching and $14.05 \mathrm{~m}$ for hard map-matching. Therefore, from this results, our proposal represents a valid technique that improves the accuracy of the $\mathrm{PF}$ even in critical scenarios, e.g. when the vehicle is skidding off the road.

\section{CONClusion}

In this paper we implemented a data fusion framework for tracking of road users applying recursive Bayesian techniques. We proposed and validated a novel soft-map matching algorithm, which is able to track users even when they are not following the road, e.g., emergency situations which are not considered in previous works. Moreover, we achieve a $45 \%$ reduction in positioning error in the considered scenarios when map-matching is not applied. Future work will be devoted to the optimization of the PF in terms of computational complexity and to the investigation of specific dynamic models for vehicles, bikers and pedestrians.

\section{ACKNOWLEDGMENT}

This work has been performed in the framework of the H2020 project 5GCAR co-funded by the EU. The comments expressed here are of the authors and do not necessarily represent the project view. The consortium is not liable for any use that may be made of any of the information contained therein.

\section{REFERENCES}

[1] C. McShane, The Automobile: A Chronology of Its Antecedents, Development, and Impact. Routledge, 1997.

[2] "Word healt organization," http://www.who.int, 2015.

[3] A. Mogelmose, M. M.Trivedi, and T. B. Moeslund, "Vision-based traffic sign detection and analysis for intelligent driver assistance systems: Perspectives and survey," IEEE Transactions on Intelligent Transportation Systems, December 2012.

[4] E. Kaplan and C. Hegarty, Understanding GPS: Principles and Applications. Artech-House, 2006.

[5] J. C. McCall and M. M. Trivedi, "Video-based lane estimation and tracking for driver assistance: Survey, system, and evaluation," IEEE Transaction on Iintelligent Transporation Systems, March 2006.

[6] F. Perez-Cruz and C. K. L. . H. Huang, "Blade: A universal, blind learning algorithm for toa localization in nlos channels," Globecom Workshops (GC Wkshps), December 2016.

[7] J. S. Pyo, D. H. Shin, and T. K. Sung, "Development of a map matching method using the multiple hypothesis technique," IEEE Proceeding of Intelligent Transportation Systems, August 2001.

[8] M. A. Quddus, R. B. Noland, and W. Ochieng, "A high accuracy fuzzy logic based map matching algorithm for road transport," Journal of Intelligent Transportation Systems, Jannuary 2007.

[9] G. Stienne, S. Reboul, M. Azmani, J. B. Choquel, and M. Benjelloun, "A multi-temporal multi-sensor circular fusion filter," Journal of Information Fusion, July 2014.

[10] A. U. Peker, O.Tosun, and T. Acarman, "Particle filter vehicle localization and map-matching using map topology," IEEE 4th Symposium on Intelligent Vehicles, July 2011.

[11] N. Bergman, Recursive Bayesian Estimation - Navigation and Tracking Applications. Linkoping, 1999.

[12] M. Arulampalam, S. Maskell, N. Gordon, and T. Clapp, "A tutorial on particle filters for online nonlinear/non-gaussian bayesian tracking," IEEE Transactions on Signal Processing, August 2002.

[13] A. Houenou, P. Bonnifait, V. Cherfaoui, and W. Yao, "Vehicle trajectory prediction based on motion model and maneuver recognition," IEEE/RSJ International Conference on Intelligent Robots and Systems (IROS), January 2014.

[14] R. Schubert, E. Richter, and G. Wanielik, "Comparison and evaluation of advanced motion models for vehicle tracking," IEEE 11th International Conference on Information Fusion, September 2008.

[15] S. J. Julier and J. K. Uhlmann, "Unscented filtering and nonlinear estimation," IEEE Proceeding, November 2004.

[16] “Open street map," https://www.openstreetmap.org, 2018.

[17] J. W. Choi and G. H. Elkaim, "Bézier curve for trajectory guidance," November 2008. 